\title{
Extramedullary haematopoiesis: An uncommon posterior mediastinal mass
}

\author{
E Marchiori, MD, PhD; M M Barreto, MD, PhD; B Hochhegger, MD, PhD; G Zanetti, MD, PhD
}

Department of Radiology, Rio de Janeiro Federal University, Rio de Janeiro, Brazil

Corresponding author: E Marchiori (edmarchiori@gmail.com)

\begin{abstract}
A 59-year-old man presented with anaemia and complaints of abdominal pain, weight loss, fatigue and dizziness. Computed tomography (CT) scans of the chest revealed bilateral paravertebral masses. The CT features were typical of extramedullary haematopoiesis (EMH). EMH should be strongly considered in a patient with bilateral, well-marginated, paravertebral thoracic masses and a history of chronic, severe anaemia.
\end{abstract}

S Afr J Rad 2013;17(3):114-115. DOI:10.7196/SAJR.870

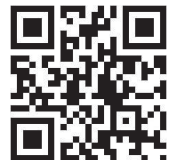

A 59-year-old man presented with anaemia and complaints of abdominal pain, weight loss, fatigue and dizziness. He had undergone a cholecystectomy due to cholelithiasis 3 years previously, and had a history of sickle-cell disease since the age of 10 years. Chest radiography depicted bilateral posterior mediastinal masses (Fig. 1). Chest computed tomography (CT) scans revealed well-marginated and lobulated bilateral paravertebral masses in the lower half of the thorax, with heterogeneous density owing to areas of fat and softtissue attenuation, suggesting long-standing disease (Fig. 2). The lung parenchyma showed no abnormality. The CT features were typical of extramedullary haematopoiesis (EMH), defined as the development and growth of haematopoietic tissue outside the bone marrow.

\section{Discussion}

EMH is seen in a variety of haematological disorders, including severe haemolytic anaemias (thalassaemia, sickle-cell anaemia, spherocytosis), leukaemia, lymphoma, myelodysplasia and myelofibrosis. Extensive replacement of normal bone marrow occurs when production is insufficient to meet the body's demands. ${ }^{[1-3]}$ The most common sites of EMH are the liver, spleen and lymph nodes, although it can occur in any organ. Thoracic involvement is less frequent, usually manifesting as bilateral lobulated masses in the lower paravertebral areas. ${ }^{[3,4]}$ The destruction of adjacent ribs and vertebrae is not seen in EMH.

The erythropoietic masses are usually asymptomatic, although the presence of EMH within the spinal canal may be associated with spinal cord compression and neurological deficit related to the level of involvement. ${ }^{[2]}$ Laceration of the mass may result in spontaneous rupture and massive haemothorax. ${ }^{[1,3]}$ Another uncommon thoracic complication is involvement of the pulmonary interstitium that can cause acute and rapidly fatal respiratory failure, ${ }^{[5,6]}$ and the formation of pleural masses that usually are asymptomatic but, rarely, develop haemothorax. ${ }^{[6,7]}$

The radiographic manifestations of thoracic EMH are unilateral or bilateral, sharply circumscribed, often lobulated, paraspinal softtissue masses. CT usually shows well-defined, lobulated, soft-tissue masses in the distal thoracic paraspinal region, which may or may not enhance after intravenous contrast administration. ${ }^{[3]}$ CT can be helpful in detecting areas of fat attenuation within these lesions and in depicting bony changes related to haematological disorders, such as thalassaemia and sickle-cell anaemia. ${ }^{[4]} \mathrm{CT}$ can also demonstrate splenic infarcts with focal calcifications or a small and dense calcified spleen (autosplenectomy), which can aid the differential diagnosis. ${ }^{[1]}$ MRI may show intermediate signal intensity on both Tl- and T2-weighted images. The masses are moderately enhancing on a postcontrast T1-weighted image. Inactive lesions show high signal intensity on both Tl- and T2-weighted MR images owing to fatty infiltration or low signal intensity on both T1- and T2-weighted MR images owing to iron deposition. ${ }^{[8]}$ Bone marrow scintigraphy with Tc-99m-labelled colloid usually reveals uptake in the paravertebral masses. ${ }^{[8]}$

The diagnosis of EMH can be established with reasonable certainty on the basis of characteristic radiological findings in a patient with a predisposing haematological condition. ${ }^{[4]}$ Invasive diagnostic 


\section{PICTORIAL INTERLUDE}

procedures are potentially hazardous because of the highly vascular nature of the thoracic masses and the haemorrhagic potential of the condition. ${ }^{[3]}$ Microscopic examination shows well-formed haematopoietic tissue. ${ }^{[1]}$

Main considerations in the differential diagnosis of EMH are tumours of the posterior mediastinum, including neurogenic masses, lymphoma, paravertebral abscesses, extrapleural cysts, lateral meningoceles, mediastinal lymph node hyperplasia, and primary and metastatic malignant neoplasms. ${ }^{[1]}$

In conclusion: EMH should be strongly considered in a patient with bilateral, well-marginated, paravertebral thoracic masses and a history of chronic severe anaemia.

1. Marchiori E, Escuissato DL, Irion KL, et al. Extramedullary hematopoiesis: Findings on computed tomography scans of the chest in 6 patients. J Bras Pneumol 2008;34(10):812-816
2. Chiam QL, Lau KK. Extramedullary haematopoiesis in thalassaemia major causing spinal cord compression. Australas Radiol 2007;51(2):168-171. [http://dx.doi.org/10.1111/j.1440-1673.2007.01689.x]

3. Pornsuriyasak P, Suwatanapongched T, Wangsuppasawad N, Ngodngamthaweesuk M, Angchaisuksiri P Massive hemothorax in a beta-thalassemic patient due to spontaneous rupture of extramedullary hematopoietic masses: Diagnosis and successful treatment. Respir Care 2006;51(3):272-276.

4. Fielding JR, Owens M, Naimark A. Intrathoracic extramedullary hematopoiesis secondary to B12 and folate deficiency: CT appearance. J Comput Assist Tomogr 1991;15(2):308-310. [http://dx.doi. 101097/00004728-199103000-00023]

5. Weinschenker P, Kutner JM, Salvajoli JV, et al. Whole-pulmonary low-dose radiation therapy in agnogenic myeloid metaplasia with diffuse lung involvement. Am J Hematol 2002;69(4):277-280. [http:// dx.doi.org/10.1002/ajh.10075]

6. Yusen RD, Kollef MH. Acute respiratory failure due to extramedullary hematopoiesis. Chest 1995;108(4):1170-1172. [http://dx.doi.org/10.1378/chest.108.4.1170]

7. Kupferschmid JP, Shahian DM, Villanueva AG. Massive hemothorax associated with intrathoracic extramedullary hematopoiesis involving the pleura. Chest 1993;103(3):974-975. [http://dx.doi.org/10.1378/ chest.103.3.974

8. Kakite S, Tanabe Y, Kinoshita F, Harada H, Ogawa T. Clinical usefulness of In-111 chloride and Tc-99m Sn colloid scintigraphy in the diagnosis of intrathoracic extramedullary hematopoiesis. Ann Nucl Med 2005;19(4):317-320. [http://dx.doi.org/10.1007/BF02984625]

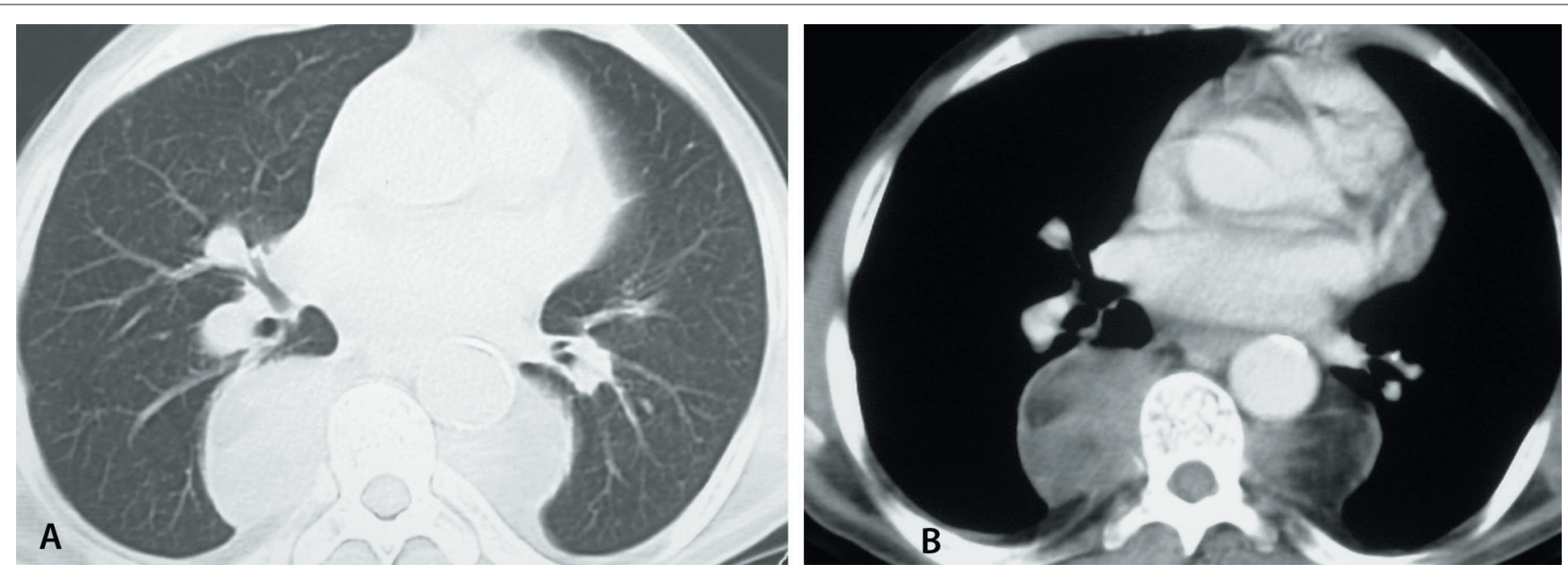

Fig. 1. Chest X-rays in frontal (A) and lateral (B) views revealed bilateral posterior lobulated retrocardiac masses. Pulmonary parenchyma and bone structures were normal.
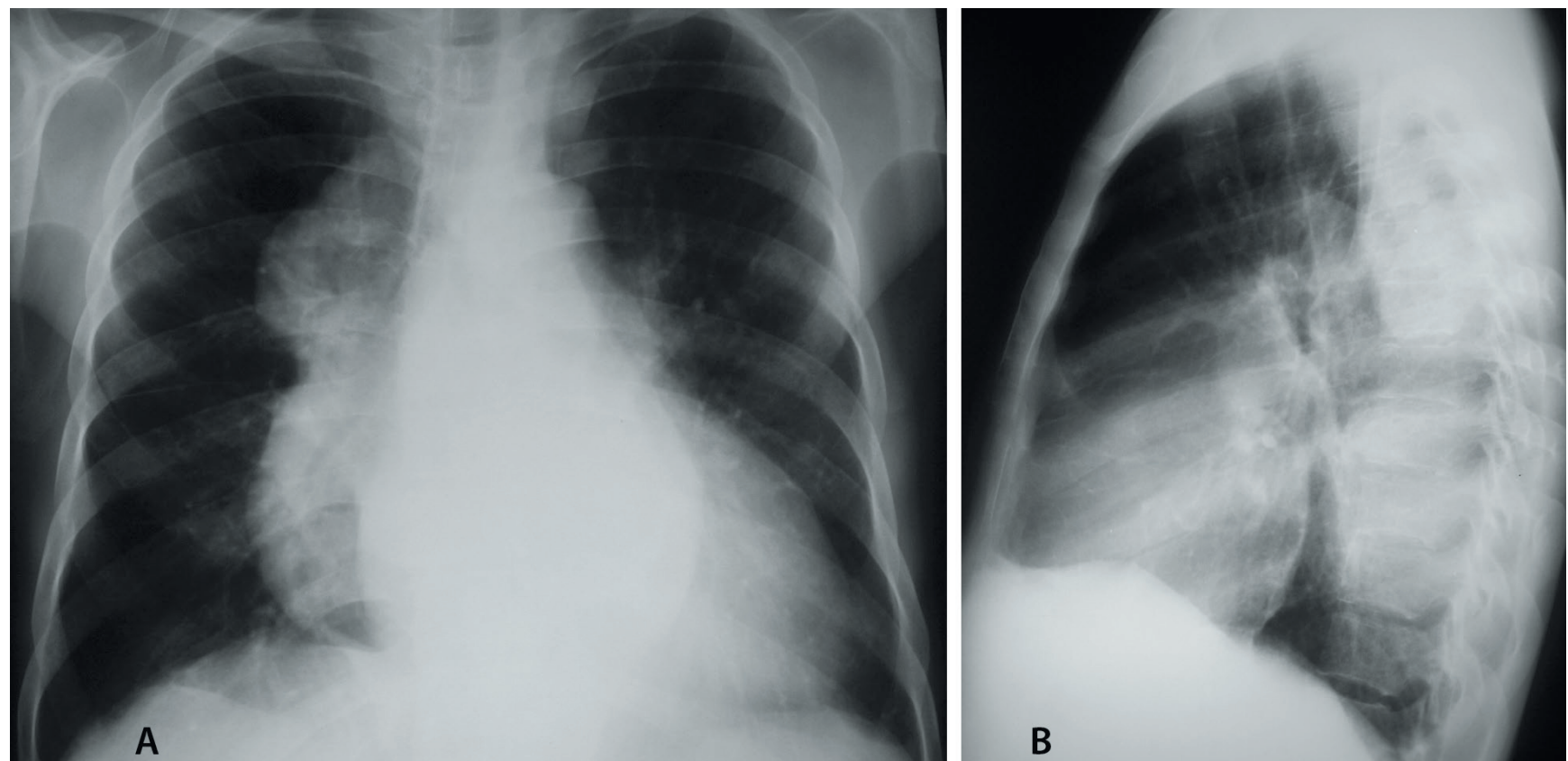

Fig. 2. Computed tomography scans with lung window (A) and mediastinal window (B) settings show bilateral masses in the inferior paravertebral thoracic regions. The masses were heterogeneous, with low-density areas suggesting a fatty component. 